\title{
Evolution de la consommation de maïs au cours du gavage et incidence sur la production de foie gras chez le canard de Barbarie
}

\author{
R. BABIlE, A. AUVERGNE, P. DELPECH *, O. MEIRIEU \\ Laboratoire de Zootechnie et des Productions Animales, \\ Ecole Nationale Supérieure Agronomique, \\ 145, Avenue de Muret, F 31076 Toulouse Cedex \\ * Laboratoire de Zootechnie, I.N.A. Paris-Grignon, \\ Centre de Recherches de Paris-Grignon, F 78850 Thiverval-Grignon
}

\begin{abstract}
Résumé
Trois lots de canards de Barbarie mâles, $(n=21,28,20)$ sont gavés au maïs pendant 17 jours. La consommation individuelle est enregistrée deux fois par jour. Le poids d'entrée en gavage (PEG), le poids saigné plumé (PSP) et le poids de foie gras (PF) sont enregistrés. Il ressort une liaison significative entre le poids de foie et la consommation totale $(0,720)$. La répartition des animaux suivant 6 classes de poids de foie permet de hiérarchiser les critères zootechniques. A l'issue d'une modélisation, la consommation est décomposée en une droite de régression représentant la phase ascendante de la consommation jusqu'à 21 gavages environ et une droite moyenne horizontale représentant la phase de finition. Ce modèle qui explique $41 \mathrm{p} .100$ des variations du poids de foie gras produit permet une meilleure connaissance du déroulement du gavage et une discrimination précoce des animaux.
\end{abstract}

Mots clés : Consommation, modélisation, foie gras, gavage, canard.

\section{Introduction}

La variabilité des performances en matière de production de foie gras résulte de nombreux facteurs. L'influence du gaveur a déjà été soulignée à plusieurs reprises dans les différentes espèces de palmipèdes (Blum \& LeclercQ, 1970 ; Mallard, 1975 ; Babile et al., 1980). Bielinska \& Bielinski (1971) ont observé chez l'oie une liaison entre la consommation globale de maïs et le poids de foie gras produit. Ils s'opposent sur ce point aux travaux antérieurs (FLEURET, 1954). Cette expérimentation a pour objet d'étudier l'évolution de la consommation de maïs par le canard de Barbarie mâle au cours du gavage et d'estimer l'incidence de ce paramètre sur les performances zootechniques.

\section{Matériel et méthodes}

\section{A. Animaux et mesures}

Trois séries de canards de Barbarie mâles $(n=21,28$ et 20$)$ ont été gavés au maïs, en épinettes, par le même gaveur. Les élevages d'origine et l'âge exact des animaux ne 
sont pas contrôlés. Dans chacune des séries, les animaux sont bagués et pesés le jour de l'entrée en gavage (PEG). Un contrôle individuel de l'ingestion de maïs cuit est réalisé à chaque gavage ( 2 fois par jour). Un échantillon de maïs cuit est prélevé quotidiennement pour mesurer la teneur en matière sèche. L'abattage a lieu après 17 jours de gavage. Le poids saigné plumé (PSP) et le poids de foie gras (PF) sont relevés.

\section{B. Analyse des données}

Les échantillons étudiés ont été répartis en 6 classes de poids de foie $(<240 \mathrm{~g}, 241$ à $350 \mathrm{~g}, 351$ à $410 \mathrm{~g}, 411$ à $510 \mathrm{~g}, 511$ à $610 \mathrm{~g},>610 \mathrm{~g}$ ). Les résultats zootechniques sont présentés en fonction de la série et de la classe à l'issue d'une analyse de variance suivant un modèle mixte (la série étant l'effet aléatoire). Les moyennes des effets sont comparées par le test de Newman \& Keuls. Les coefficients de corrélation entre les différents paramètres sont estimés.

L'évolution de la consommation en fonction du nombre de gavages est évaluée dans un premier temps, série par série, par régression linéaire, en comparant les pentes des différentes classes de foie, puis individuellement une modélisation est proposée à partir de ces résultats. Les courbes type résultantes sont composées d'une part d'une droite de régression linéaire, et d'autre part d'une droite horizontale. La droite de régression est définie par $\mathrm{y}=\mathrm{a}+\mathrm{bx}$ où :

$\mathrm{y}=$ la consommation de matière sèche pour un gavage

$a=$ l'ordonnée à l'origine $\left(A_{0}\right)$;

$\mathrm{b}=$ la pente (Bpente) de la droite de régression ;

$\mathrm{x}=$ le numéro du gavage considéré.

Les paramètres de cette droite sont déterminés selon la méthode du modèle «bent stick $\gg$ (Morris, 1983), où le point culminant $(\mathrm{N})$ de la droite de régression est fixé pour une somme des carrés des écarts résiduels minimum.

La droite horizontale (Yplateau) représente la valeur moyenne autour de laquelle oscille la consommation en fin de gavage (après le point $\mathrm{N}$ ). Une analyse en régression multiple permet d'évaluer la valeur du modèle. Comme pour les critères zootechniques, une analyse de variance sert à tester les différents critères du modèle $\left(\mathrm{A}_{\omega}\right.$, Bpente, Yplateau, N).

\section{Résultats - Discussion}

Le tableau 1 présente les performances zootechniques moyennes en fontion de la série et de la classe de foie. La série 1 malgré un PEG nettement inférieur aux deux autres (3070 g contre $3579 \mathrm{~g}$ et $3573 \mathrm{~g}$ ) obtient un poids moyen de foie gras satisfaisant $(440 \mathrm{~g})$. L'absence de différence significative sur ce critère est liée à la variabilité des résultats. Ceci confirme l'importance du gaveur et de l'élevage des animaux (Simon et al., 1976; Babile et al., 1985). Les animaux de la série 1, rationnés sévèrement en fin de période d'élevage, ont une prise de poids et une consommation de mais plus importantes $(P<0,01)$. De ce fait, les rendements en foie (PF sur PSP) apparaissent très significativement supérieurs $(\mathrm{P}<0,001)$. 


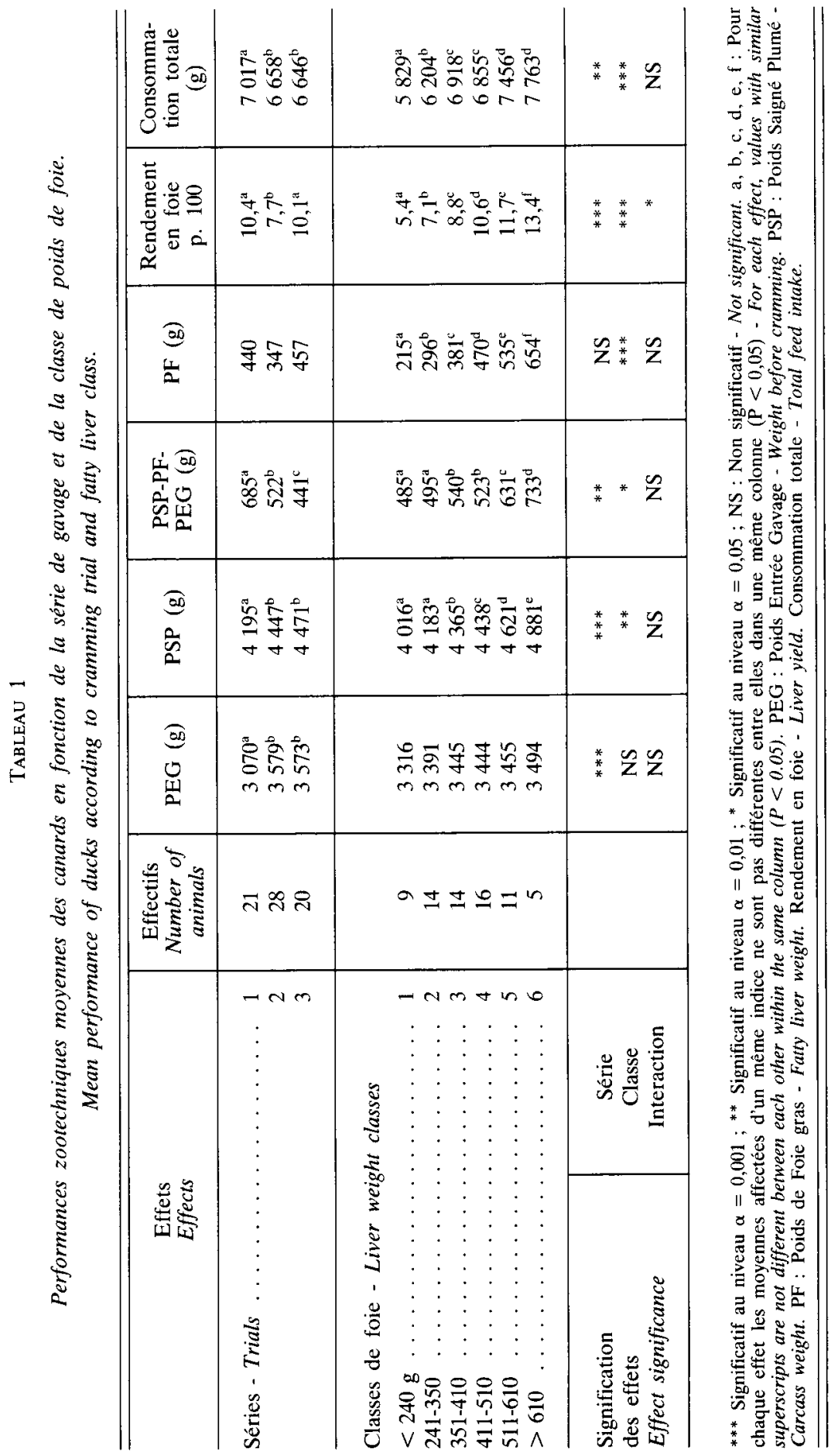


Des effets significatifs $(\mathrm{P}<0,05)$ à très significatifs $(\mathrm{P}<0,001)$ apparaissent pour le PSP, et la consommation en fonction du classement des foies gras. Il en est de même pour l'engraissement général des animaux estimé grossièrement (PSP - PF - PEG) en l'absence de la mesure du poids vif au moment de l'abattage. La faible liaison $(r<0,2)$ entre PEG et PF (BABILE et al., 1980) est confirmée par l'absence d'effet classe sur le PEG. Les animaux produisant un foie dans la classe $1(<240 \mathrm{~g})$ ont tendance à être plus légers à l'entrée du gavage et présentent de même la consommation et les performances d'abattage les plus faibles. A l'opposé, les animaux des classes 5 et 6 (>511 g) consomment des quantités équivalentes mais se distinguent significativement par leurs performances. Les classes intermédiaires $(2,3$, et 4$)$, significativement différentes des précédentes $(1,5$ et 6$)$, se comportent distinctement selon les paramètres mesurés. Pour la classe 2 présentant des foies gras déclassés (241 à $350 \mathrm{~g}$ ), la prise de poids et la consommation sont significativement inférieures à celles des deux autres classes (3 et 4). Malgré un PEG et une consommation identiques, les classes 3 et 4 présentent des écarts de poids de foie très importants. Les causes de ces écarts peuvent être multiples comme par exemple des antécédents d'élevages (BABILE et al., 1985) ou la variabilité dans le potentiel génétique des animaux (BABILE et al., 1980). L'interaction significative qui apparaît sur le rendement de foie résulte du fait que dans la série 2 les performances des classes 3, 4 et 5 sont inférieures à celles des autres séries.

Les liaisons positives (tabl. 2) entre la consommation totale et, le PEG $(0,426)$, le PSP $(0,639)$, le gain de poids $(0,481)$, le poids de foie gras $(0,721)$, sont à rapprocher des résultats de Bielinska \& Bielinski (1971) sur l'oie $(0,549$ avec le PEG, 0,797 avec

TABLEaU 2

Corrélations entre la consommation totale de matière sèche et les performances de production du canard de Barbarie gavé.

Correlations between total feed intake and performance of crammed Muscovy duck.

\begin{tabular}{|c|c|c|c|c|c|c|}
\hline & $\begin{array}{l}\text { Effectifs } \\
\text { Number of } \\
\text { animals }\end{array}$ & PEG & PSP & PSP-PEG & $\begin{array}{c}\text { PSP- } \\
\text { (PEG-PF) }\end{array}$ & $\mathrm{PF}$ \\
\hline $\begin{array}{lll}\text { Série } \ldots . & 1 \\
& & 2 \\
& & 3\end{array}$ & $\begin{array}{l}20 \\
25 \\
20\end{array}$ & $\begin{array}{l}0,211 \\
0,581^{* *} \\
0,402\end{array}$ & $\begin{array}{l}0,406 \\
0,696^{* *} \\
0,740^{* *}\end{array}$ & $\begin{array}{l}0,310 \\
0,358 \\
0,726^{* *}\end{array}$ & $\begin{array}{r}-0,148 \\
0,149 \\
0,320\end{array}$ & $\begin{array}{l}0,627^{* *} \\
0,749^{* *} \\
0,763^{* *}\end{array}$ \\
\hline$x^{2}$ & & $1,96 \mathrm{NS}$ & $2,67 \mathrm{NS}$ & $3,86 \mathrm{NS}$ & $2,00 \mathrm{NS}$ & $1,32 \mathrm{NS}$ \\
\hline $\begin{array}{l}\text { Coefficient } \\
\text { moyen } \ldots . . \\
\text { Mean } \\
\text { coefficient } \\
\text { Intervalle de } \\
\text { confiance ..... } \\
\begin{array}{l}\text { Confidence } \\
\text { interval }\end{array}\end{array}$ & 65 & $\begin{array}{c}0,426^{* * *} \\
0,191 / 0,615\end{array}$ & $\begin{array}{c}0,639^{* * *} \\
0,458 / 0,769\end{array}$ & $\begin{array}{c}0,481^{* * *} \\
0,256 / 0,656\end{array}$ & \begin{tabular}{|c|}
$0,114 \mathrm{NS}$ \\
$-0,149 / 0,361$
\end{tabular} & $\begin{array}{c}0,721^{* * *} \\
0,570 / 0,825\end{array}$ \\
\hline
\end{tabular}




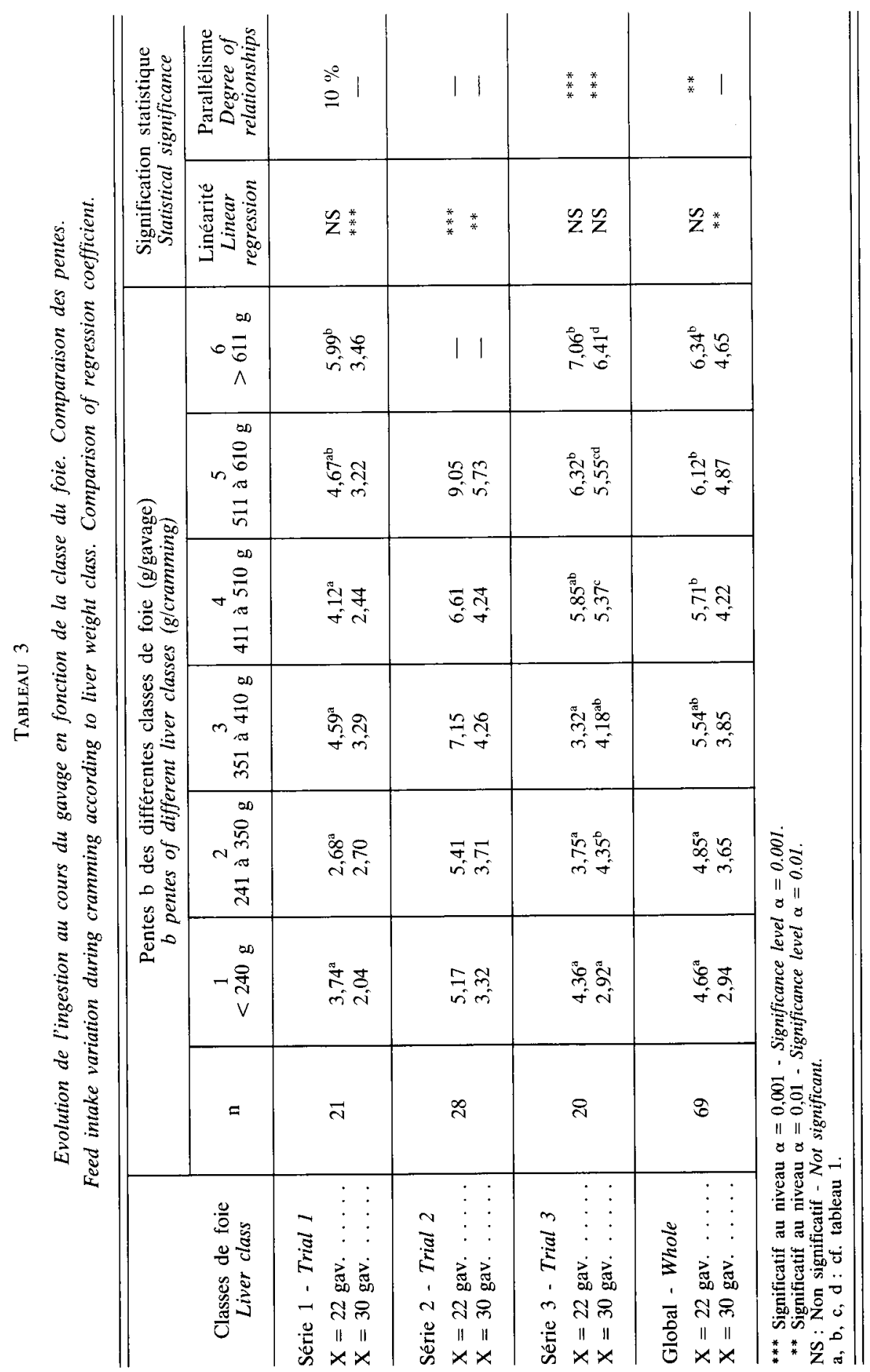


le gain de poids et 0,693 avec le PF). Cette corrélation élevée entre la consommation totale de maïs et le poids de foie gras produit, suggère que des différences d'évolution du niveau d'ingestion pourraient expliquer la variabilité des performances.

Des tests d'ajustement préalables montrent que quelle que soit la classe de foie, l'évolution de la consommation en fontion du nombre de gavages n'est en général linéaire que jusqu'à 22 gavages (tabl. 3). La régression n'est plus linéaire en raison des variations de consommation après ce stade. La série 3 poursuit cependant une progression linéaire jusqu'à 30 gavages, alors que l'absence de linéarité observée pour la série 2 provient de la variabilité des consommations individuelles et semble liée aux médiocres résultats enregistrés dans ce lot.

La comparaison des pentes deux à deux montre que l'on peut dans tous les cas faire deux groupes de droites avec une zone de chevauchement qui peut se situer dans des classes différentes selon la série (entre 351 et 410 g pour l'ensemble des animaux).

Les différences de pente et le point de rupture à 22 gavages, observés au travers de ces résultats justifient l'utilisation du modèle "bent stick " qui permet de caractériser une phase ascendante et linéaire de la prise de maïs et une autre plus irrégulière représentée par une droite horizontale correspondant à la phase de finition des animaux (fig. 1). Ce modèle permet de définir quatre paramètres principaux caractérisant l'évolution de la consommation individuelle au cours du gavage : la capacité d'ingestion de chaque animal à l'entrée en gavage $\left(A_{0}\right)$, la vitesse de progression de la distribution de maîs (Bpente), le numéro du gavage $(\mathrm{N})$ correspondant à la fin de la phase ascendante du gavage, et le niveau moyen d'ingestion au cours de la phase de finition (Yplateau).

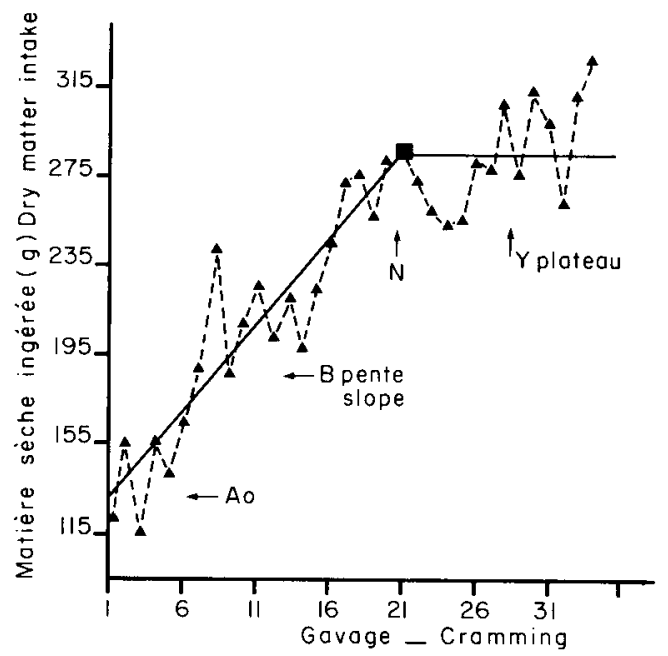

FIG. 1

Modélisation de la consommation individuelle.

Régression de I à $N$ gavages. Consommation $=A_{o}+B\left(N^{*}\right.$ du gavage $)$.

Yplateau $=$ ingestion moyenne entre $N+1$ et la fin du gavage.

Individual feed intake pattern. Regression from 1 to $N$ crammings.

Feed intake $=A_{o}+B$ (cramming number).

Yplateau $=$ mean ingestion between $N+1$ and end of cramming.

Exemple ci-dessus $\left(A_{o}=121,39 ; B=77,88 ; N=21 ;\right.$ Yplateau $=284,39 ; r$ de la régres$\operatorname{sion}=0,926)$. 
Comme pour les performances zootechniques, ces paramètres sont étudiés en fonction de la série et de la classe de foie (tabl. 4). Les différences significatives qui apparaissent, concernant l'ordonnée à l'origine $\left(A_{0}\right)$, traduisent surtout les disparités de capacité d'ingestion, consécutives aux antécédents d'élevage des animaux. Bien que gavés par la même personne, on ne peut exclure l'importance du facteur humain dans les écarts observés. La pente significativement plus élevée de la série 2 correspond à une progression moyenne des consommations individuelles jusqu'à 22 gavages, avec cependant de grandes disparités entre les animaux, ce qui est confirmé par l'absence de linéarité de la régression globale pour cette série. Malgré l'absence de différence significative entre les niveaux moyens du plateau (Yplateau), il ressort que celui de la série 3, qui a réalisé les plus gros foies gras $(457 \mathrm{~g})$, est le plus élevé. A l'opposé, une pente forte associée à un plateau plus bas pour la série 2 est le signe d'une sousconsommation en fin de gavage, qui a eu pour conséquence une médiocre production de foie gras $(347 \mathrm{~g})$. L'allongement de la phase ascendante des deux dernières séries $(\mathrm{N}=22$ gavages), peut marquer l'absence de préparation spécifique, ce qui correspond à des capacités d'ingestion réduites en début de gavage. Les animaux de la série 1, qui présentent un niveau d'ingestion plus élevé, ont la phase ascendante la plus courte ( $\mathrm{N}=17$ gavages).

\section{TABleau 4}

Effets série et classe de foie sur les critères définis par le modèle.

Effects of trial and liver weight class on model criteria.

\begin{tabular}{|c|c|c|c|c|c|}
\hline & $\underset{\mathbf{n}}{\text { Effectifs }}$ & $A_{0}(g)$ & $\begin{array}{c}\text { Bpente } \\
\text { (g/gavage) }\end{array}$ & Yplateau (g) & $\begin{array}{c}\mathrm{N} \\
\left(\mathrm{N}^{0} \text { de gav. }\right)\end{array}$ \\
\hline \multicolumn{6}{|l|}{ Séries - Trials } \\
\hline$\ldots \ldots$ & 21 & $149,7^{\mathrm{a}}$ & $5,24^{4}$ & 233,3 & $17,2^{\mathrm{a}}$ \\
\hline 2 & 28 & $123,9^{b}$ & $6,38^{\mathrm{b}}$ & 229,0 & $22,4^{\mathrm{b}}$ \\
\hline $3 \ldots \ldots$ & 20 & $109,6^{\mathrm{c}}$ & $5,67^{\mathrm{a}}$ & 253,3 & $22,3^{\mathrm{b}}$ \\
\hline \multicolumn{6}{|l|}{$\begin{array}{l}\text { Classes de foie } \\
\text { Liver weight classes }\end{array}$} \\
\hline$\ldots$ & 9 & 116,1 & $5,49^{a}$ & $194,4^{\mathrm{a}}$ & 21,0 \\
\hline $2 \ldots \ldots \ldots \ldots \ldots$ & 14 & 126,3 & $4,79^{\mathrm{a}}$ & $225,6^{\mathrm{b}}$ & 21,1 \\
\hline$\ldots \ldots \ldots$ & 14 & 133,6 & $5,97^{\mathrm{b}}$ & $235,2^{b}$ & 21,1 \\
\hline 4 & 16 & 125,9 & $5,90^{\mathrm{b}}$ & $247,4^{c}$ & 21,4 \\
\hline 5 & 11 & 129,8 & $6,82^{\mathrm{b}}$ & $260,4^{c}$ & 19,1 \\
\hline 6 & 5 & 135,9 & $7,39^{\mathrm{b}}$ & $270,2^{c}$ & 20,2 \\
\hline \multicolumn{6}{|l|}{$\begin{array}{l}\text { Signification des effets } \\
\text { Effect significance }\end{array}$} \\
\hline$\ldots \ldots \ldots \ldots$ & & $* * *$ & ** & $10 \%$ & $* * *$ \\
\hline Classes & & NS & * & $* *$ & NS \\
\hline Inter & & NS & NS & NS & NS \\
\hline
\end{tabular}

Légende : cf. tableau $\mathrm{n}^{\circ} 1$.

$A_{w}$, Bpente, $Y, N$ : cf. figure 1 . 


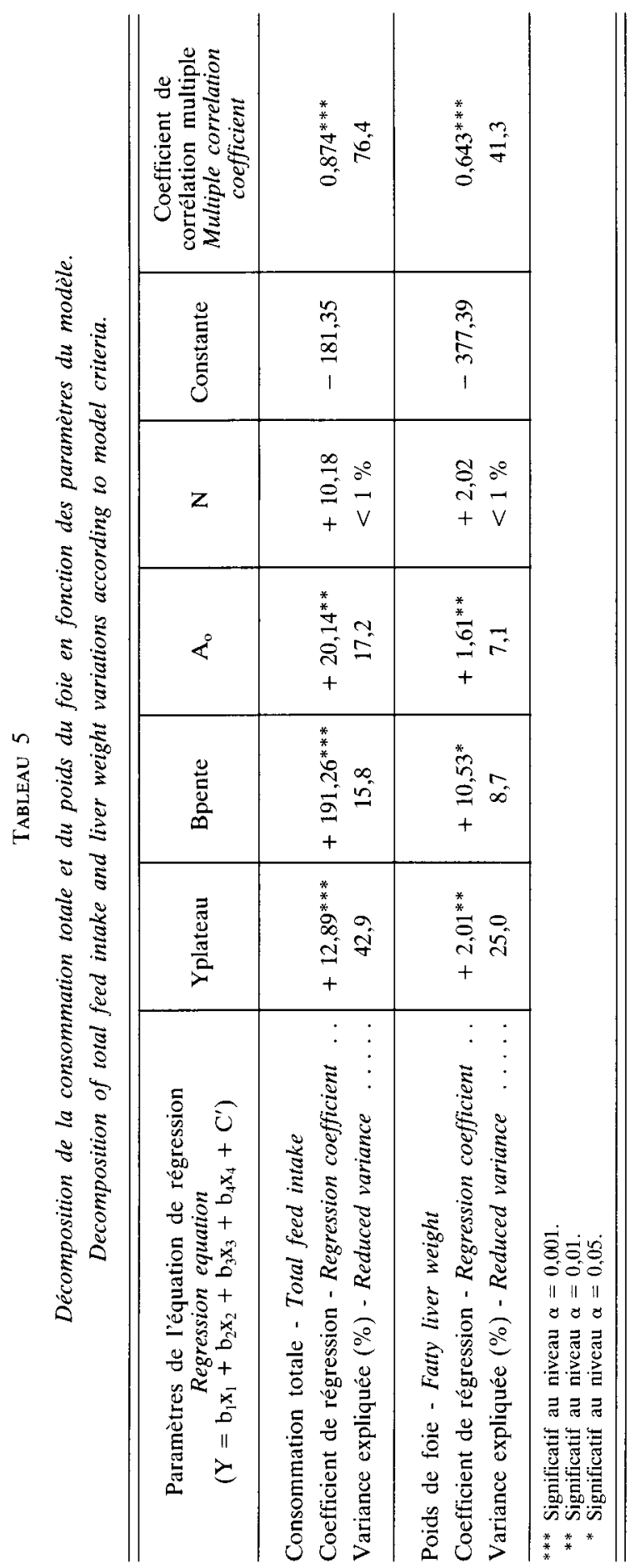


Les classes de foie gras ne sont pas discriminées par le niveau de consommation au début du gavage, alors que la pente et surtout le niveau du plateau diffèrent significativement en fonction de ce critère. Tous les animaux ayant produit moins de $350 \mathrm{~g}$ de foie présentent une pente de consommation inférieure aux autres; pour les plus performants ( $>511 \mathrm{~g}$ de foie) les pentes sont plus élevées, bien que non significativement différentes de celles des classes intermédiaires (351 à $510 \mathrm{~g})$. Les niveaux moyens de plateau augmentent avec le poids de foie gras, bien que trois groupes seulement soient identifiables sur le plan statistique (Classe 1: $194 \mathrm{~g}$; Classes 2 et $3: 230 \mathrm{~g}$ et Classes 4 , 5 et $6: 255 \mathrm{~g}$ ). L'absence d'interaction entre le lot de gavage et la classe confirme la validité du modèle utilisé.

Quarante et un pour cent $(41,3$ p. 100) des variations de poids de foie sont expliqués par les paramètres du modèle (tabl. 5), le plateau expliquant à lui seul 25 p. 100 de la variation. Le coefficient de corrélation $(0,643)$ entre le poids de foie et l'ensemble de ces paramètres est assez proche de celui obtenu sur l'ensemble des lots avec la consommation totale $(0,653)$. Le modèle utilisé traduit correctement la consommation totale $(76,4$ p. 100 de la variance : c'est le plateau qui est le plus représentatif $(42,9$ p. 100 de la variance $)$.

\section{Conclusion}

La décomposition de la distribution de maïs au cours du gavage en deux périodes (phase ascendante et phase de finition) caractérisées par quatre paramètres $\left(A_{0}\right.$, Bpente, $\mathrm{N}$ et Yplateau), permet de mieux appréhender l'incidence des variations biquotidiennes de la consommation sur la production de foie gras (fig. 2).

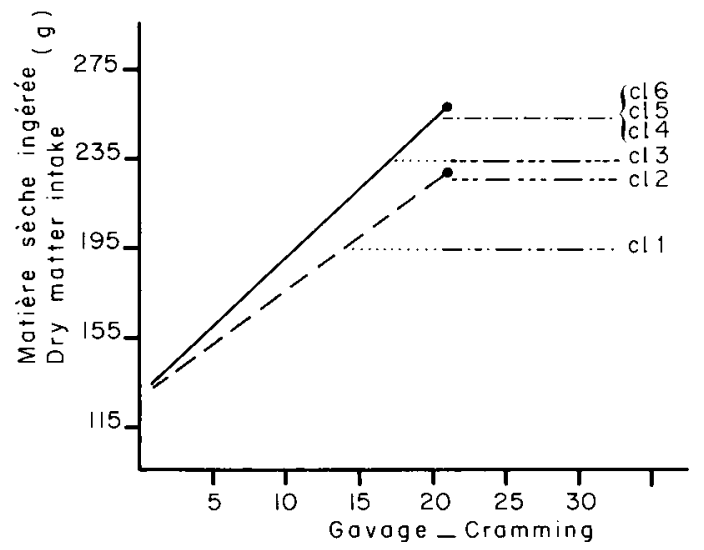

FIG. 2

Différents types de courbe de consommation en fonction des paramètres moyens du modèle.

Different feed intake curves according to average parameters of the model.

Les pentes et les plateaux représentés par les mêmes symboles ne diffèrent pas significativement entre eux $(\mathrm{P}<0,05)$ - Regression coefficients and Yplateau with the same graphic symbol are not significantly different $(P<0.05)$. 
L'influence du niveau d'ingestion en début de gavage fait ressortir l'intérêt d'une préparation spécifique des canards au cours de l'élevage. L'étude de la progression de la prise alimentaire au cours de la phase ascendante met en évidence deux types de pentes, l'une faible ( $+5,1 \mathrm{~g}$ par gavage), qui ne peut conduire qu'à des performances mauvaises à médiocres $(<350 \mathrm{~g})$, l'autre plus forte $(+6,3 \mathrm{~g}$ par gavage $)$ correspondant aux meilleurs résultats $(>350 \mathrm{~g})$.

La phase de finition représentée par le "Yplateau " est la plus importante puisqu'elle explique à elle seule 25 p. 100 de la variation des poids de foie gras produits. Un niveau de plateau faible (194 g) correspond à des canards ayant vraisemblablement un handicap, depuis le début du gavage, lié à leurs antécédents d'élevage (Pour la classe 1: $\mathrm{PEG}=3316 \mathrm{~g}: \mathrm{A}_{\mathrm{o}}=116 \mathrm{~g} ; \mathrm{B}=5,49 \mathrm{~g}$ par gavage). Les foies gras les plus lourds $(>411 \mathrm{~g})$ résultent d'animaux ayant un haut niveau d'ingestion en phase de finition. Pour ces derniers, tout le gavage a été bien conduit (Bpente et Yplateau élevés). Les classes intermédiaires (foies gras entre 241 et $410 \mathrm{~g}$ ) ont le même niveau de plateau ( $230 \mathrm{~g}$ en moyenne), mais se distinguent par des phases ascendantes différentes. Une progression faible de la consommation en début de gavage, ne permet pas d'atteindre un bon plateau et, a pour conséquence la production d'un poids de foie médiocre (241 à $350 \mathrm{~g}$ ). Une consommation maximale, observée en fin de phase ascendante et correspondant au gavage $\mathrm{N}(\mathrm{N}=21$ en moyenne), très supérieure au niveau du plateau, traduit une chute de consommation en période de finition. La production de foie gras moyenne (entre 350 et $410 \mathrm{~g}$, classe 3) est en partie la conséquence de ce phénomène.

Cette décomposition de la consommation de maïs pendant le gavage permet une prédiction correcte du poids de foie gras. Le modèle utilisé, n'expliquant que 41 p. 100 des variations du poids de foie, facilite cependant l'analyse du déroulement du gavage et, dans une certaine mesure, celle des performances techniques observées.

Reçu en juillet 1986. Accepté en octobre 1986.

\title{
Remerciements
}

Nous remercions, M. Claude Legrendre qui a réalisé le gavage et M. Briere pour son aide dans la conception du modèle.

\author{
Summary \\ Maize feed intake variation during cramming and its effect \\ on fatty liver production of Muscovy ducks
}

Three groups of Muscovy ducks $(\mathrm{n}=21,28,20)$ were crammed with maize for 17 days. Individual feed intake was recorded twice a day. Weight before cramming (PEG), carcass weight (PSP) and fatty liver weight were measured. A significant relationship was observed between liver weight and total feed intake $(r=0.720$ ) (tabl. 2). Performance traits were ranked thanks to the distribution of ducks into 6 liver weight classes. 
Using a mathematical model, feed intake was decomposed into a regression line ( $y=A_{o}+B x$, where $y$ is the dry matter intake per cramming and $x$ the cramming number) which represents the increase in feed intake until about 21 crammings and a mean straight line which represents the finishing stage ("Yplateau ") (fig. 1). This model characterized two mean slopes (Bpente) and three main plateaus (Yplateau) (tabl. 4). The different combinations allowed to discriminate four groups : class 1 , class 2 , class 3 and classes 4,5 , and 6 (fig. 2). The last three classes showed a progressive increase in all the parameters of the model. This model explained $40 \mathrm{p} .100$ of fatty liver weight variance and facilitated the understanding of cramming process and an early discrimination of ducks.

Key words : Feed intake, modelling, fatty liver, cramming, duck.

\section{Références bibliographiques}

Babile R., Matheron G., Delpech P., Farran M.T., 1980. La production de foie gras de canard de Barbarie mâle : analyse des différents facteurs de variation. Ann. Zootech., 29, 265-277.

Babile R., Auvergne A., Delpech P., 1985. Influence d'un rationnement précoce sur les performances de gavage de différents types génétiques de canards. Colloque "la génétique du canard de Barbarie (Cairina moschata) » INRA Toulouse-Auzeville. 15-16 octobre 1985.

Bielinska K., Bielinski K., 1971. Intensive fattening of geese for fat livers. Rocz Nauk Roln B93-4, 55-64.

Blum J.C., Leclerce B., 1970. Facteur favorable à la formation du foie gras chez l'oie : la distribution d'un régime enrichi en choline pendant la période précédant le gavage. Ann. Zootech., 19, 347-351.

Fleuret P.H., 1954. La production du foie gras de volaille destiné à la consommation. Physiologie, Pathologie, Chimie et Cytologie des Foie Gras, 24, 25, 26 mars 1953, 97-131, C.N.R.S. Ed.

Mallard Y., 1975. Etude des performances de gavage chez l'oie. Mémoire de fin d'études. E.N.S.A. Toulouse, p. 40.

Morris T.R., 1983. The interpretation of response data from animal feeding trials. In HaresigN W. Recent Advance in animal nutrition, 13-23, Butterworths Ed.

Simon J., Rousselot-Pailley D., Monachon G., Blum J.C., 1976. Influence du rationnement de l'oie landaise pendant la période d'élevage sur les performances : croissance, production de foie gras par gavage et reproduction. $V^{c}$ Congrès Avicole Européen. Malte, W.P.S.A. vol. 1, 483493. 